\title{
Energy Efficient DF Relay Placement in $\alpha-\mu$ Fading Channel with Cooperative and Non-Cooperative Schemes
}

\author{
Biswajit GHOSH ${ }^{1}$, Sankalita BISWAS ${ }^{2}$, Aniruddha CHANDRA ${ }^{3}$, Ashis Kumar MAL ${ }^{2}$ \\ ${ }^{1}$ IT Depr., Future Institute of Engineering \& Management, 700150 Kolkata, India \\ ${ }^{2}$ ECE Dept., National Institute of Technology, 713209 Durgapur, India \\ ${ }^{3}$ Dept. of Radio Electronics, Brno University of Technology, 61600 Brno, Czech Republic
}

biswanerist@gmail.com, sanky.lita1@gmail.com, chandra@feec.vutbr.cz,akmal@ece.nitdgp.ac.in

Manuscript received March 04, 2016

\begin{abstract}
In this paper we examine the energy efficiency of relay aided wireless sensor network (WSN) links where the two-hop transmission between source and destination is realized with an adaptive decode and forward (DF) relay. The receiver may use only the relayed signal (non-cooperative mode) or it may be combined with the signal directly available from the source (co-operative mode). In the second mode, we have considered two combining schemes, selection combining (SC) and maximum ratio combining (MRC). A generalized $\alpha-\mu$ model is used to characterize fading and spectrally efficient $M$-ary quadrature amplitude modulation (MQAM) is chosen as the modulation technique. The $\alpha-\mu$ model is quite general in nature and it encompasses other popular fading models such as Rayleigh and Nakagami-m. The result of our analysis reveals that combining does not necessarily help in reducing energy requirement and the decision of whether 'to use' or 'not to use' combining depends on the source to destination distance. Further, a comparison between MRC and SC from the energy efficiency perspective manifests that using MRC does not automatically guarantee maximum energy savings and the SC strategy is more robust against parameter variations as well as incurs lower receiver complexity. We also investigate the optimal relay location, which minimizes energy requirement, for all the above mentioned relaying strategies, and study how different system parameters affect the optimal relay placement.
\end{abstract}

\section{Keywords}

$\alpha-\mu$ fading channel, energy efficiency, maximum ratio combining, relay placement, selection combining

\section{Introduction}

Wireless sensor networks (WSNs) has proliferated into diverse sectors, healthcare, surveillance, logistics, and environmental monitoring. Enhancing WSN lifetime has been always a huge motivation for the researchers [1]. Also, from the commercial point of view, the rapidly expanding worldwide wireless market demands smarter energy solutions as the energy costs comprise a large share of operational expenditures $(\mathrm{OpEx})$. Cooperation among sensor nodes is one of the key ideas to minimize the energy consumption, and in its simplest form, may be realized by finding an intermediate relay node $\left(R_{n}\right)$ between the source node $\left(S_{n}\right)$ and the destination node $\left(D_{n}\right)$. Cooperative diversity helps to mitigate the effects of channel fading by combining the message received through direct path with the signal received through relayed path. However, cooperation increases receiver complexity at the destination node, and it is important to investigate whether and how much energy can be saved with cooperation under different circumstances.

In this paper, we considered two diversity schemes, selection combining (SC) and maximum ratio combining (MRC), and provided the respective calculations for outage probabilities and energy consumptions. These cooperative techniques are then compared with the non-cooperative mode, i.e. when the destination ignores the direct path signal available from the source. The system under study uses an adaptive decode-and-forward (DF) relay [2] which saves energy by refraining from transmission in the event of link outage and employs $M$-ary quadrature amplitude modulation (MQAM) technique that achieves high rate transmission without increasing the bandwidth. To make our analysis compatible with different realistic scenarios, we have modeled the small-scale fading with $\alpha-\mu$ statistics. The $\alpha-\mu$ model [3], [4] can be considered as a generalized fading statistics and includes many other statistical models like onesided Gaussian, Rayleigh, and the Nakagami- $m$, as special cases. For example, when $\alpha=2$, the model reduces to simple Nakagami- $m$ distribution, with the other parameter $\mu$ being equal to $m[3]$.

The current text is an extension of our earlier work [5-7] where energy efficiency of non-cooperative relayed transmission was studied for the Rayleigh fading model. In this paper, we compare the non-cooperative scheme with diversity based cooperative schemes and present our analysis for generalized 
$\alpha-\mu$ fading model. The basic energy calculation framework is adopted from [8]. During comparison, we first find analytical expressions for energy consumption per successful bit transmission (in microJoule) for all the schemes and investigate optimal relay placement location on the sourcerelay-destination $\left(S_{n}-R_{n}-D_{n}\right)$ line. Next, we examine how different design parameters such as source to destination distance $(D)$, path loss exponent $(a)$, target end-to-end outage probability $(O)$, spectral efficiency $(R)$, and fading parameters $(\alpha$ and $\mu$ ) affect the energy efficiency and optimal relay location. In this regard, we would like to cite some relevant literature to clarify the novelty of our work. The authors in [9] investigate two-dimensional optimal relay placement on the basis of energy efficiency without considering any general fading model. Some directions regarding energy efficient relay placements in multi-hop Rayleigh channel were mentioned in [10]. In [11], [12] the authors described energy efficiency of some cooperative and non-cooperative transmission schemes in WSNs over Nakagami- $m$ fading channel, but nothing related to relay placement was mentioned. In [13], transmit power is minimized by establishing an acceptable limit for the packet loss whereas the authors in [14] assessed the performance of DF relay with parallel coding and MRC. On the other hand, most of the papers related to generalizing fading models do not address the issue of energy efficiency with relaying. For example, the authors in [15] present exact closed-form of probability density function and cumulative distribution function of generalized $\alpha-\mu$ fading model, and also investigate the performance of equal-gain combining and maximal-ratio combining. Again, in [16] the authors discuss the performance of SC diversity and effect of severe fading condition based on $\eta-\mu$ fading channels. To the best of our knowledge, the energy efficiency of relayed communication over generalized fading model has been so far discussed only in [17] and [18]. The $\eta-\mu$ model, which characterizes non-homogeneity of the diffused scattering field, is considered in [17]. The $\alpha-\mu$ model is used for characterizing non-linearity of the propagation medium and the respective energy calculations are presented in [18]. However, the goal of [18] was to assess how introduction of simple SC diversity serves beneficial over non-cooperative scheme from the energy perspective. In this paper, we compare energy efficiency performance of simple SC with the complex MRC technique over relayed $\alpha-\mu$ channel.

The paper is organized as follows. Section 2 presents the energy consumption models. In Sec. 3 numerical results are discussed. Finally, Sec. 4 concludes the paper.

\section{Energy Consumption Modeling}

The total power consumption of a wireless sensor node can be divided into two parts, circuit power consumption and transmit power consumption. For short range transmission, we can express the total power consumption as:

$$
P_{\mathrm{C}}=P_{\mathrm{PA}}+P_{\mathrm{TX}}+P_{\mathrm{RX}}
$$

where $P_{\mathrm{PA}}$ indicates power consumption by the power amplifier (PA) at transmitter (TX) while power for the rest of transmitter blocks are given by $P_{\mathrm{TX}}$, and $P_{\mathrm{RX}}$ denotes the power consumption by circuit components at receiver (RX). For detailed expressions of $P_{\mathrm{TX}}$ and $P_{\mathrm{RX}}$, the readers are referred to [5] and [6]. As per the notational conventions in [7],

$$
P_{\mathrm{PA}}=(\xi / \eta) \times \Psi \times E_{\mathrm{b}} \times 2 R_{\mathrm{b}}
$$

where $\Psi=(4 \pi / \lambda)^{2} D^{a} M_{l} N_{f} / G$. In (2), $\eta$ is the drain efficiency of the PA and $\xi$ is the peak-to-average power ratio (PAPR), which for MQAM can be written as, $\xi=$ $3(\sqrt{M}-1) /(\sqrt{M}+1)$, with $M$ being the modulation order [11]. The parameters $D, a, \lambda, M_{l}, N_{f}$, and $G$ denote distance between source and destination, path loss exponent, carrier wavelength $\left(\lambda=c / f_{\mathrm{c}} ; c=\right.$ speed of light in free space, $f_{\mathrm{c}}=$ carrier frequency), link margin, noise figure and combined TX-RX antenna gain, respectively. The received power can be expressed as, $P_{\mathrm{R}}=E_{\mathrm{b}} \times R_{\mathrm{b}}$, where $E_{\mathrm{b}}$ is the required energy per bit at receiver and $R_{\mathrm{b}}$ is the bit rate.

Fading reduces the signal to noise ratio (SNR) available at the receiver. If we consider an $\alpha-\mu$ fading channel, the probability density function (PDF) of the faded signal envelope follows the distribution [4]:

$$
f_{R}(r)=\frac{\alpha \mu^{\mu} r^{\alpha \mu-1}}{\bar{r}^{\alpha \mu} \Gamma(\mu)} \exp \left(-\mu \frac{r^{\alpha}}{\bar{r}^{\alpha}}\right)
$$

where $\bar{r}=\sqrt{\mathcal{E}\left(R^{2}\right)} ; \mathcal{E}(\cdot)$ denotes the expectation operator, $\Gamma(z)=\int_{0}^{\infty} t^{z-1} \exp (-t) \mathrm{d} t$ is the gamma function, and $\alpha>0$, $\mu>0$ are fading parameters. A detailed description of how the small scale fading parameters $(\alpha, \mu)$ affect the channel statistics is available in [4].

An outage occurs when the received SNR at the receiver falls below a threshold $\beta$ and this outage probability may be derived from (3) as [15]:

$$
O=\operatorname{Pr}\{\gamma \leq \beta\}=\frac{\Gamma\left(\mu, \frac{\mu \beta^{\alpha}}{\bar{\gamma}^{\alpha}}\right)}{\Gamma(\mu)}
$$

where $\Gamma(\cdot, \cdot)$ denotes incomplete gamma function defined as $\Gamma(z, y)=\int_{y}^{\infty} t^{z-1} \exp (-t) \mathrm{d} t$, and $\bar{\gamma}$ is average channel SNR. The SNR outage threshold $\beta$, which allows error free decoding, may be obtained from Shannon's capacity theorem [19]

$$
\beta=2^{R}-1
$$

where $R\left(R=R_{\mathrm{b}} / B ; B\right.$ is the bandwidth) is the spectral efficiency. For a given maximum allowable outage value, the required average $\mathrm{SNR}$ at the receiver end, $\bar{\gamma}$, may be found from (4), and from the calculated average SNR we can find the required bit energy $\left(E_{\mathrm{b}}\right)$. This, in turn, may be used to find $P_{\mathrm{PA}}$, the power consumption of the power amplifier, according to (2), and the total energy consumption per bit is

$$
E_{\mathrm{bt}}=P_{\mathrm{C}} / R_{\mathrm{b}}=\left(P_{\mathrm{PA}}+P_{\mathrm{TX}}+P_{\mathrm{RX}}\right) / R_{\mathrm{b}}
$$




\subsection{Non-Cooperative Communication}

When the destination receives the signal only via an adaptive DF relay and ignores any direct signal available from the source, an outage occurs either if there is an outage in the $S_{n}-R_{n}$ link (the DF relay do not forward signal any further in this case) or there is no outage in the $S_{n}-R_{n}$ link but outage occurs in the $R_{n}-D_{n}$ link. If we express the outage probability along $S_{n}-R_{n}$ link by $O_{S R}$ and in the $R_{n}-D_{n}$ link as $O_{R D}$, the outage probability at destination is simply $O_{\mathrm{R}}=O_{S R}+\left(1-O_{S R}\right) O_{R D}$. For similar fading severities in both the links, i.e. $O_{S R}=O_{R D}=O_{\mathrm{r}}$, the end-to-end outage probability along the entire relayed path is given by:

$$
O_{\mathrm{R}}=O_{\mathrm{r}}+\left(1-O_{\mathrm{r}}\right) O_{\mathrm{r}}=2 O_{\mathrm{r}}-O_{\mathrm{r}}^{2}
$$

where $O_{\mathrm{r}}=\left.O\right|_{\bar{\gamma}=\bar{\gamma}_{R}}$ is the outage probability between each pair of nodes in the relayed path. The parameter, $O_{\mathrm{R}}$, represents the percentage of time when energy consumption does not contribute to successful transmission. From (4) we can write,

$$
O_{\mathrm{R}}=2 \frac{\Gamma\left(\mu, \frac{\mu \beta^{\alpha}}{\bar{\gamma}_{R}^{\alpha}}\right)}{\Gamma(\mu)}-\left[\frac{\Gamma\left(\mu, \frac{\mu \beta^{\alpha}}{\bar{\gamma}_{R}^{\alpha}}\right)}{\Gamma(\mu)}\right]^{2} .
$$

One may find the minimal bit energy required for the non-cooperative scheme:

$$
E_{\mathrm{b}, \text { relay }}=N_{0} \times \bar{\gamma}_{R}
$$

from the required average SNR, $\bar{\gamma}_{R}$, which satisfies (8) for an overall target outage probability, $O_{\mathrm{R}}$. In (9), $N_{0}$ is the one-sided noise power spectral density.

To transmit one bit through the relayed path, the respective energy consumption is given by:

$$
\begin{aligned}
E_{\mathrm{bt}, \text { relay }}= & O_{\mathrm{r}}\left(\frac{P_{\mathrm{PA}, S R}+P_{\mathrm{TX}}+P_{\mathrm{RX}}}{R_{\mathrm{b}}}\right) \\
& +\left(1-O_{\mathrm{r}}\right) \\
& \times\left(\frac{P_{\mathrm{PA}, S R}+P_{\mathrm{PA}, R D}+2 P_{\mathrm{TX}}+2 P_{\mathrm{RX}}}{R_{\mathrm{b}}}\right)
\end{aligned}
$$

where $P_{\mathrm{PA}, S R}=P_{\mathrm{PA}, R D}=2(\xi / \eta) \Psi E_{\mathrm{b} \text {,relay }} R_{\mathrm{b}}$. Due to the adaptive nature of the relay, the relay does not forward the signal in case of an outage in the $S_{n}-R_{n}$ path which is reflected in the first term of (10), where only the energy at source $\left(P_{\mathrm{PA}, S R}\right.$ and $\left.P_{\mathrm{TX}}\right)$ and relay $\left(P_{\mathrm{RX}}\right)$ is accounted. In the case of no outage, the signal is again retransmitted from the relay and this scenario is characterized in the second term of (10) with additional power terms $\left(P_{\mathrm{PA}, R D}, P_{\mathrm{TX}}\right.$, and $\left.P_{\mathrm{RX}}\right)$.

Considering the average number of retransmissions required to compensate for outage, $1 /\left(1-O_{\mathrm{R}}\right)$, we may express the average energy consumption per bit for successful transmission over the relay path as:

$$
E_{\mathrm{bt}, \text { relay_suc }}=\frac{E_{\mathrm{bt}, \text { relay }}}{1-O_{\mathrm{R}}} .
$$

The expressions for $E_{\mathrm{bt}, \text { relay }}$ is as expressed in (10) and $O_{\mathrm{R}}$ is the target outage probability.

\subsection{Cooperation Through SC}

In a classical SC implementation all the various paths to the destination are active at the same time and the one with the highest SNR is being considered as the combined output. Thus, outage occurs at combiner output when the path with the highest SNR is in outage. To be more precise, outage at destination means all the paths are in outage at the same time, since even the path with the highest SNR has an instantaneous SNR value that is less than the outage threshold. For a single relay, the end to end outage probability with selection combining would be:

$$
\begin{aligned}
O_{\mathrm{SC}} & =O_{S R} \times O_{S D}+\left(1-O_{S R}\right) \times O_{R D} \times O_{S D} \\
& =\left(2-O_{\mathrm{s}}\right) O_{\mathrm{s}}^{2}
\end{aligned}
$$

where $O_{\mathrm{s}}=\left.O\right|_{\bar{\gamma}=\bar{\gamma}_{\mathrm{SC}}}$ is the outage probability between each pair of nodes, i.e. $O_{S R}=O_{R D}=O_{S D}=O_{\mathrm{s}}$, and, $\bar{\gamma}_{\mathrm{SC}}$, is the required average SNR with SC. From (4) we can write:

$$
O_{\mathrm{SC}}=\left[2-\frac{\Gamma\left(\mu, \frac{\mu \beta^{\alpha}}{\bar{\gamma}_{\mathrm{SC}}^{\alpha}}\right)}{\Gamma(\mu)}\right]\left[\frac{\Gamma\left(\mu, \frac{\mu \beta^{\alpha}}{\bar{\gamma}_{\mathrm{SC}}^{\alpha}}\right)}{\Gamma(\mu)}\right]^{2} .
$$

Alike the non-cooperative case, we may first set a target total outage probability, $O_{\mathrm{SC}}$, and find the average $\mathrm{SNR}$, $\bar{\gamma}_{\mathrm{SC}}$, which satisfies the value from (13). This SNR value determines the minimum bit energy required:

$$
E_{\mathrm{b}, \mathrm{SC}}=N_{0} \times \bar{\gamma}_{\mathrm{SC}} \text {. }
$$

For energy consumption calculation, we have to consider the energy consumption in all the paths, since all the paths are active at any point in time for $\mathrm{SC}$, i.e.,

$$
\begin{aligned}
E_{\mathrm{bt}, \mathrm{SC}}= & O_{\mathrm{s}}\left(\frac{P_{\mathrm{PA}, S R}+P_{\mathrm{TX}}+2 P_{\mathrm{RX}}}{R_{\mathrm{b}}}\right) \\
& +\left(1-O_{\mathrm{s}}\right) \\
& \times\left(\frac{P_{\mathrm{PA}, S R}+P_{\mathrm{PA}, R D}+2 P_{\mathrm{TX}}+3 P_{\mathrm{RX}}}{R_{\mathrm{b}}}\right)
\end{aligned}
$$

where $P_{\mathrm{PA}, S R}=P_{\mathrm{PA}, R D}=2(\xi / \eta) \Psi E_{\mathrm{b}, \mathrm{SC}} R_{\mathrm{b}}$, with $E_{\mathrm{b}, \mathrm{SC}}$ as specified in (14). It may be noted that the second term in (15) contains a power term $3 P_{\mathrm{RX}}$ in contrast to $2 P_{\mathrm{RX}}$ in (10). The extra receiver power term accounts for the extra branch at receiver node $\left(R_{n}\right)$ for realizing diversity combining. Next, considering the retransmissions required, we may write the average energy consumption per bit for successful transmission as

$$
E_{\mathrm{bt}, \mathrm{SC} \_ \text {suc }}=\frac{E_{\mathrm{bt}, \mathrm{SC}}}{1-O_{\mathrm{SC}}}
$$

where $E_{\mathrm{bt}, \mathrm{SC}}$ is given by (15).

\subsection{Cooperation Through MRC}

The advantage of using MRC to combine the signals obtained over the relayed path and the direct path is, even if the SNR of the individual paths are below the desired threshold, 
the combined SNR $\left(\gamma_{\mathrm{MRC}}\right)$ might exceed it, as the combined SNR at the destination is summation of the individual branch SNRs. The trade-off for the improved performance is the added receiver complexity because all the available paths should be optimally combined at the receiver.

We consider the following two cases: case $A$, when there is an outage in the $S_{n}-R_{n}$ link, and case $B$, when there is not. The relay refrains from transmission under situation $A$ due to its adaptive nature. This results in only the direct $S_{n}-D_{n}$ path to be active, i.e. $\gamma_{\mathrm{MRC}}=\gamma_{D}$, where $\gamma_{D}$ represents the SNR along the direct path, and outage occurs at destination when the direct path is in outage, i.e.,

$$
O_{A}=O_{S D}=O_{\mathrm{m}}
$$

where

$$
O_{\mathrm{m}}=\left.O\right|_{\bar{\gamma}=\bar{\gamma}_{\mathrm{MRC}}}=\frac{\Gamma\left(\mu, \frac{\mu \beta^{\alpha}}{\bar{\gamma}_{\mathrm{MRC}}^{\alpha}}\right)}{\Gamma(\mu)}
$$

is the outage probability between each pair of nodes, and, $\bar{\gamma}_{\mathrm{MRC}}$, is the required average SNR with MRC.

For case $B$, there is no outage in the $S_{n}-R_{n}$ link, and both the relayed and the direct path will be active. Hence, the combined SNR is found by adding the SNRs of both the paths, i.e. $\gamma_{\mathrm{MRC}}=\gamma_{D}+\gamma_{R D}$, where $\gamma_{R D}$ is the SNR along the $R_{n}-D_{n}$ link. It may be noted here that the term $\gamma_{R D}$ should be replaced by $\gamma_{S R D}$, the end-to-end SNR, in case of an amplify-and-forward (AF) relay [20].

The corresponding outage probability, $O_{B}=$ $\operatorname{Pr}\left\{\gamma_{\mathrm{MRC}} \leq \beta_{m}\right\}=\operatorname{Pr}\left\{\gamma_{R D} \leq \beta_{m}-\gamma_{D} \mid \gamma_{D}\right\}$, is calculated as:

$$
O_{B}=\int_{0}^{\beta_{m}} \operatorname{Pr}\left\{\gamma_{R D} \leq \beta_{m}-\gamma_{D}\right\} f\left(\gamma_{D}\right) \mathrm{d} \gamma_{D}
$$

where the SNR threshold is, $\beta_{m}=2^{2 R}-1$, as the spectral efficiency is double in this case [11]. The limits of the integration are taken from 0 to $\beta_{m}$ since if $\gamma_{D}$ becomes greater than $\beta_{m}$ then the summation of $\gamma_{D}$ and $\gamma_{R D}$ can never be less than $\beta_{m}$. Using (4) and (3), the integral in (19) is expressed as:

$$
\begin{aligned}
O_{B}= & \int_{0}^{\beta_{m}} \frac{\Gamma\left(\mu, \frac{\mu\left[\beta_{m}-\gamma_{D}\right]^{\alpha}}{\bar{\gamma}_{\mathrm{MRC}}^{\alpha}}\right)}{\Gamma(\mu)} \frac{\alpha \mu^{\mu} \gamma_{D}^{\alpha \mu-1}}{\bar{\gamma}_{\mathrm{MRC}}^{\alpha \mu} \Gamma(\mu)} \\
& \times \exp \left(-\mu \frac{\gamma_{D}^{\alpha}}{\bar{\gamma}_{\mathrm{MRC}}^{\alpha}}\right) \mathrm{d} \gamma_{D}
\end{aligned}
$$

which is computed numerically due to the complex nature of the integrand.

Finally, the total outage probability for MRC, $O_{\mathrm{MRC}}=$ $O_{S R} O_{A}+\left(1-O_{S R}\right) O_{B}$, is computed as:

$$
O_{\mathrm{MRC}}=O_{\mathrm{m}} O_{A}+\left(1-O_{\mathrm{m}}\right) O_{B}=O_{\mathrm{m}}^{2}+\left(1-O_{\mathrm{m}}\right) O_{B}
$$

where, the expressions of $O_{\mathrm{m}}$, and $O_{B}$ are given by (18) and (20), respectively.

For a given target total outage probability, $O_{\mathrm{MRC}}$, it is possible to find the required average SNR, $\bar{\gamma}_{\mathrm{MRC}}$, from (21).
This SNR value determines the minimum bit energy required

$$
E_{\mathrm{b}, \mathrm{MRC}}=N_{0} \times \bar{\gamma}_{\mathrm{MRC}} .
$$

The energy consumption calculation should take care of all possible paths, and is given by:

$$
\begin{aligned}
E_{\mathrm{bt}, \mathrm{MRC}}= & O_{\mathrm{m}}\left(\frac{P_{\mathrm{PA}, S R}+P_{\mathrm{TX}}+2 P_{\mathrm{RX}}}{R_{\mathrm{b}}}\right) \\
& +\left(1-O_{\mathrm{m}}\right) \\
& \times\left(\frac{P_{\mathrm{PA}, S R}+P_{\mathrm{PA}, R D}+2 P_{\mathrm{TX}}+3 P_{\mathrm{RX}}}{R_{\mathrm{b}}}\right)
\end{aligned}
$$

where $P_{\mathrm{PA}, S R}=P_{\mathrm{PA}, R D}=2(\xi / \eta) \Psi E_{\mathrm{b}, \mathrm{MRC}} R_{\mathrm{b}}$, with $E_{\mathrm{b}, \mathrm{MRC}}$ being specified in (22). The second term in (23) also contains a power term $3 P_{\mathrm{RX}}$ similar to (15) for the reason explained in the previous subsection. The average energy consumption per bit for successful transmission in MRC, considering the retransmissions, is expressed as:

$$
E_{\mathrm{bt}, \mathrm{MRC} \_ \text {suc }}=\frac{E_{\mathrm{bt}, \mathrm{MRC}}}{1-O_{\mathrm{MRC}}}
$$

which is calculated by substituting the expression of $E_{\mathrm{bt}, \mathrm{MRC}}$ given by (23).

\section{Results and Discussions}

In this section, we compare the energy consumption for one successful bit transmission, measured in $\mu \mathrm{J}$, for noncooperative (without-combining) and cooperative (SC and MRC) schemes in a WSN with three collinear nodes. The topology is described in Fig. 1, where the relay is placed on the same line joining the source and the destination nodes. The coordinates for source, relay and destination are, $(0,0)$, $\left(d_{S R}, 0\right)$ and $(D, 0)$, respectively. The inter-node channels are characterized with the $\alpha-\mu$ fading model.

During our comparison, we consider the effect of following metrics, namely, the source to relay distance $\left(d_{S R}\right)$, source to destination distance $(D)$, and spectral efficiency $(R)$. The vertical axis of such plots denote energy consumption per bit, in $\mu \mathrm{J}$, whereas for the horizontal axis we describe the location of the relay in terms of the normalized distance, $d_{S R} / D ;\left(0 \leq d_{S R} / D \leq 1\right)$, a unit less quantity which denotes the ratio of distance between source and relay to the total distance between source and destination. This enables us to analyze the optimum location of relay irrespective of the source-destination distance $(D)$.

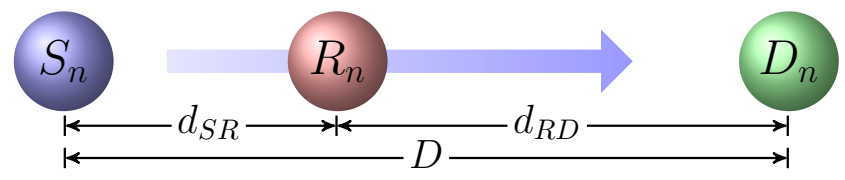

Fig. 1. Collinear relay placement. 


\begin{tabular}{lll}
\hline Parameter & Notation & Value \\
\hline Modulation order & $M$ & 16 \\
\hline Drain efficiency & $\eta$ & 0.35 \\
\hline Carrier frequency & $f_{\mathrm{c}}$ & $2.5 \mathrm{GHz}$ \\
\hline TX-RX distance & $D$ & $35 \mathrm{~m}$ \\
\hline Path loss exponent & $a$ & 3 \\
\hline Link margin & $M_{l}$ & $40 \mathrm{~dB}$ \\
\hline Noise figure & $N_{f}$ & $10 \mathrm{~dB}$ \\
\hline Antenna gain & $G$ & $5 \mathrm{dBi}$ \\
\hline Target outage & $O$ & 0.01 \\
\hline Noise PSD & $N_{0} / 2$ & $-174 \mathrm{dBm} / \mathrm{Hz}$ \\
\hline Spectral efficiency & $R$ & $2 \mathrm{~b} / \mathrm{s} / \mathrm{Hz}$ \\
\hline Bandwidth & $B$ & $10 \mathrm{kHz}$ \\
\hline
\end{tabular}

Tab. 1. System parameters [6], [11].

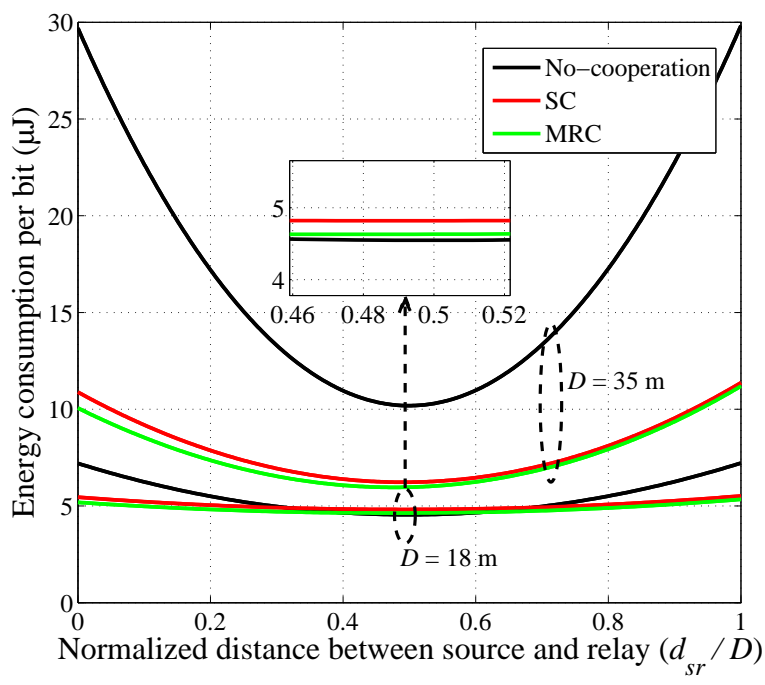

Fig. 2. Energy efficiency comparison with no-cooperation, SC, and MRC for different values of $D$.

We also investigate the optimal location of the relay for both cooperative and non-cooperative schemes and how fading parameters (i.e. $\alpha$ and $\mu$ ), target outage probability, path loss exponent, or spectral efficiency affects the optimum location. It is worth to notice that the variation of optimum location $\left(d_{S R}^{*}\right)$ with path loss exponent $(a)$ is similar in all cases; for low $a$ values the optimum location is closer to $S_{n}$, i.e. $\left(d_{S R}^{*} / D<0.5\right)$, but as $a$ increases, the optimum location converges to the mid-point of $S_{n}-D_{n}$ line $\left(d_{S R}^{*} / D \rightarrow 0.5\right)$. This fact inspired us to replace the vertical axis for all optimum location plots with path loss exponent, and depict how other parameters affect the above mentioned trend. The optimal relay locations for each path loss exponent value are found from the valley points when energy consumption expressions are plotted against normalized source to relay distance.

All the figures presented in this section are obtained by evaluating the respective analytical expressions through MATLAB. The non-cooperative scheme is omitted in some plots as the topic is already covered in [18] in detail. The power consumption of TX and RX, which are $P_{\mathrm{TX}}=97.6$ $\mathrm{mW}$ and $P_{\mathrm{RX}}=112.4 \mathrm{~mW}$, respectively, are calculated by summing up the power consumed by individual circuit components (see Tab. 1 of [6] for details) of the TX and RX blocks. All the other system parameter values are described in Tab. 1, unless otherwise specified.

\subsection{Effect of $S_{n}-R_{n}$ and $S_{n}-D_{n}$ Distances}

In Fig. 2 we compare how the energy consumption per bit changes when the relay is shifted along the $S_{n}-D_{n}$ line. The optimum location is at mid-point $\left(d_{S R}^{*} / D=0.5\right)$ for non-cooperative scheme. On the other hand, the optimum location is closer to the source $\left(d_{S R}^{*} / D<0.5\right)$ when combining at the destination is realized.

Also, as seen from Fig. 2, when the $S_{n}-D_{n}$ distance is $18 \mathrm{~m}$ (or less), the non-cooperative scheme is more energy efficient than the SC and MR schemes, if we consider that the relay is placed at an optimum location in both instances. Thus, we may conclude that for very short distances non-cooperative scheme is a better choice than the cooperative schemes. The situation is reversed for $D=35 \mathrm{~m}$, or for more than $18 \mathrm{~m}$ in general. Among the two combining schemes, MRC scheme is more energy efficient compared to SC scheme.

\subsection{Effect of Target Outage Probability}

It was observed earlier [18] that if we allow worser target outage, non-cooperative strategy may outperform the combining schemes. We do not repeat such a plot as the energy saving is nominal and the phenomenon is observed only at such a high outage probability that the link can be rendered as 'broken' for realistic communication purposes.

Instead we compare the optimal location of the relay for different end-to-end outage values in Fig. 3. We see that for high outage values (lines with markers) the optimal location of the relay is shifted towards the source node. Again, for a given outage value (lines with different colors), the shift is nominal when combining is not used, more in case of SC, and most prominent for MRC.

\subsection{Effect of Spectral Efficiency $(R)$}

When we compare the energy consumed per bit for different spectral efficiency values (see Fig. 4), we found that MRC performs marginally better with the performance being identical when the relay is close to the destination. The energy consumption is of course higher when a higher spectral efficiency is required.

Figure 5 shows how the optimal location of the relay is shifted when $R$ or $a$ decreases. Similar to Fig. 3, the shift is more for the MRC scheme when compared to the SC scheme. Further, the optimal location becomes fixed for higher values of spectral efficiency $(R \geq 4)$. This indicates that the optimal location is affected by the spectral efficiency when its value is low. The same trend was seen for the no-combining scheme as well (not shown in figure). 


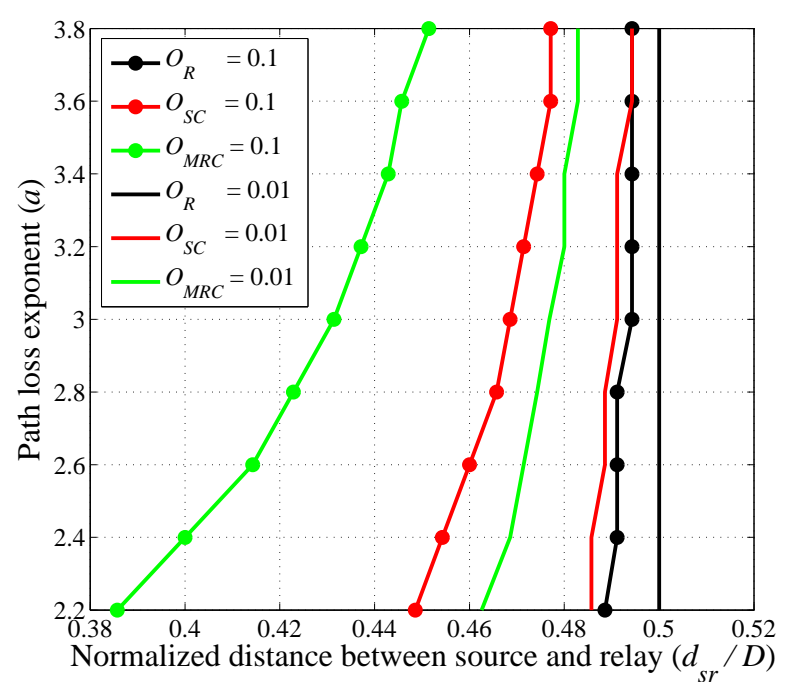

Fig. 3. Effect of target outage probability on optimal location of the relay.

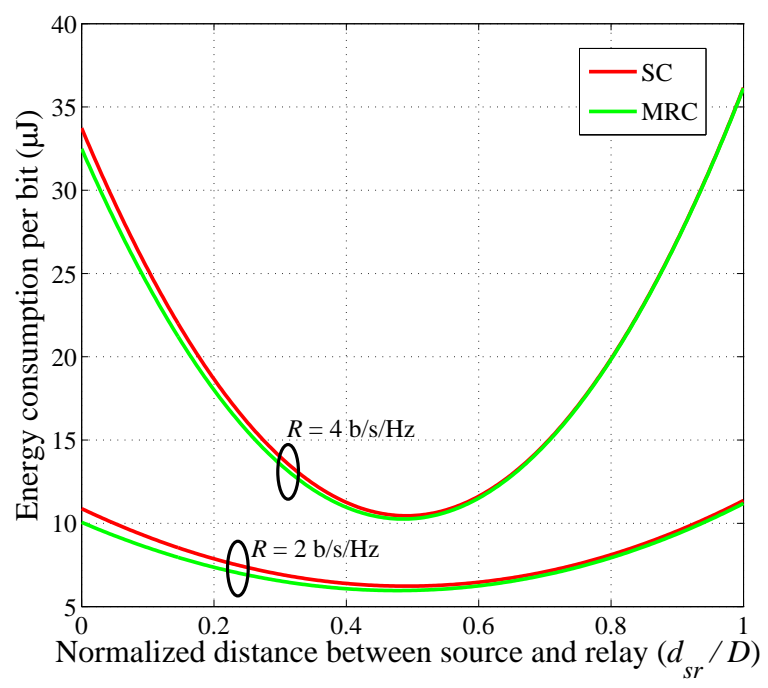

Fig. 4. Energy efficiency comparison between SC and MRC for different values of $R$ (spectral efficiency).

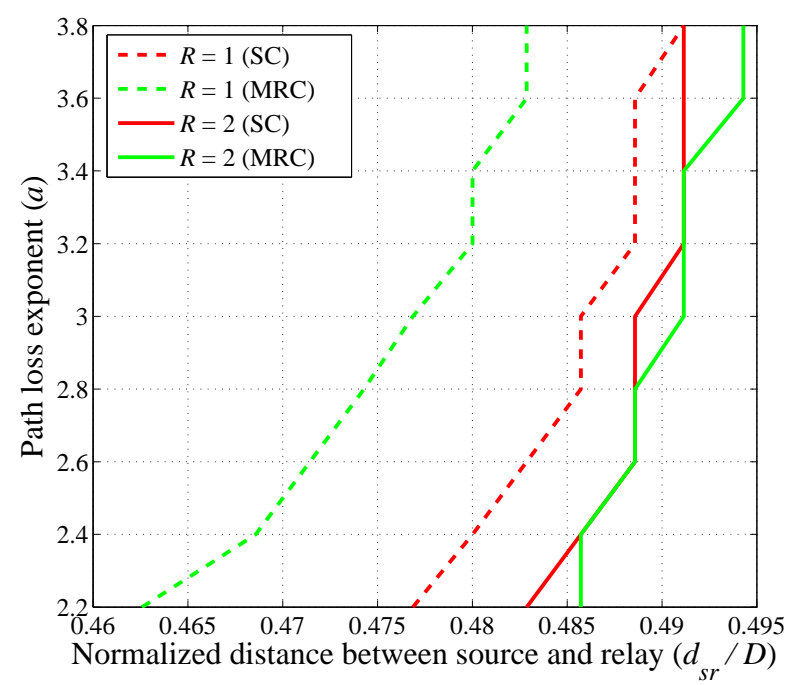

Fig. 5. Effect of spectral efficiency on optimal location of the relay.

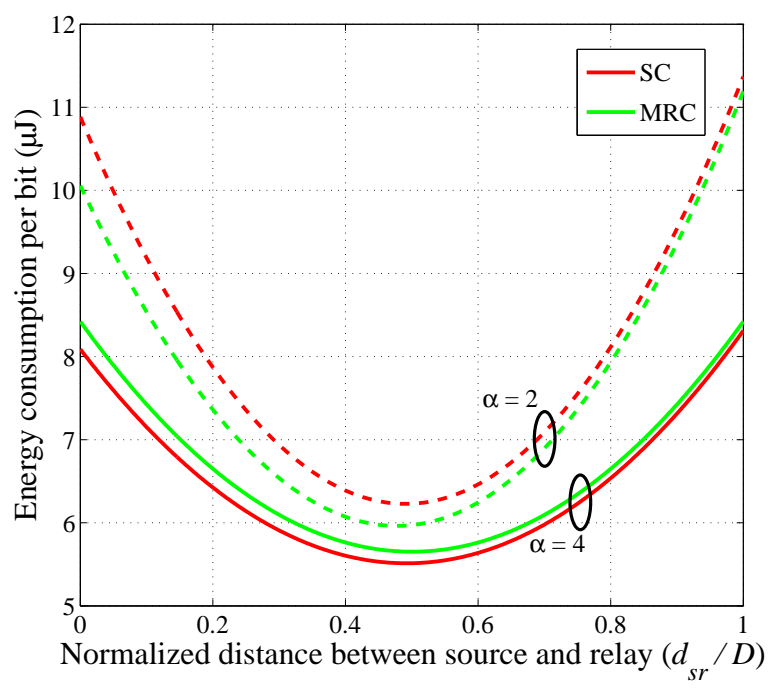

Fig. 6. Energy efficiency comparison between SC and MRC for different values of $\alpha(\mu=1)$.

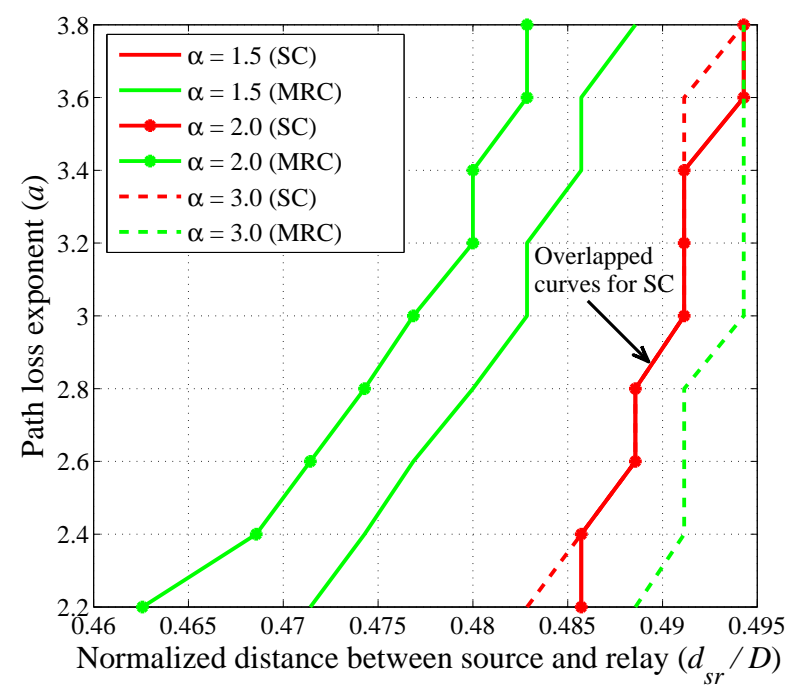

Fig. 7. Effect of $\alpha$ on optimal location of the relay $(\mu=1)$.

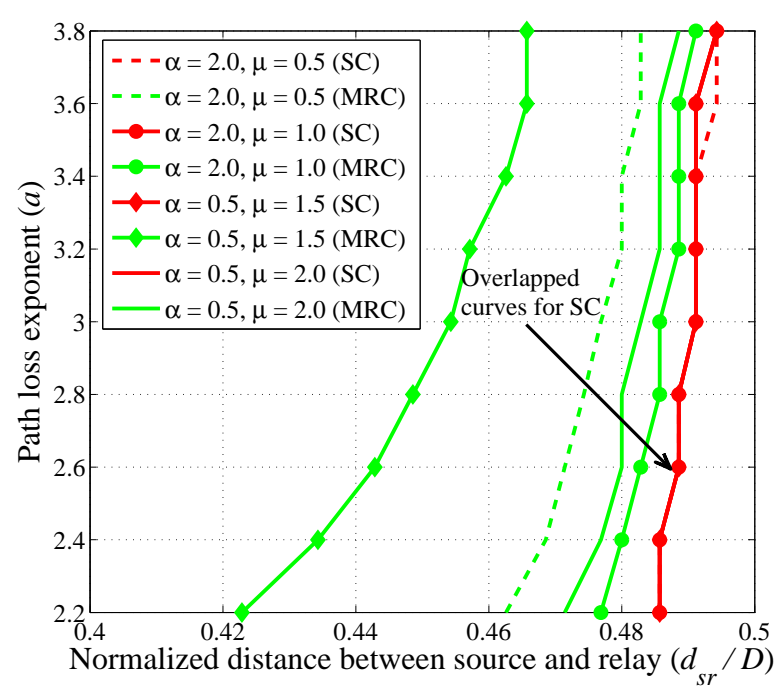

Fig. 8. Effect of $\mu$ on optimal location of the relay $(\alpha=0.5,2)$. 


\subsection{Effect of Fading Parameters $(\alpha, \mu)$}

The fading severity changes with fading parameters ( $\alpha$ and $\mu$ ) of the model. As we change $\alpha$ and $\mu$ values, the required average SNR at receiver also changes so that the end to end outage probability can be maintained.

Let us consider the effect of $\alpha$ first. Figure 6 shows that for $\mu=1$ if we increase value of $\alpha$ from 2 to 4 , the energy requirement reduces for both SC and MRC. However, the most interesting observation is that while MRC is more energy efficient for $\alpha=2$, for $\alpha=4$ SC outperforms MRC from the energy efficiency perspective.

Figure 7 shows that for $\mu=1$, if we increase $\alpha$ value from 1.5 to 3 , there is a significant change of optimal relay location when MRC is employed. The effect is almost non-existent for SC, and the curves for different $\alpha$ values overlap.

Next, we study the effect of $\mu$ in Fig. 8, keeping the value of $\alpha$ fixed at 0.5 and 2. The curves for SC overlap, irrespective of the fading parameter values. The variation with path loss exponent is also very low for SC. This is another interesting finding because if SC is employed and locations of all three nodes remain fixed, the energy efficiency do not change with a change of fading environment (either small scale fading parameters or the large scale fading loss exponent), as the optimal location is not affected much. For MRC, the variation is quite noticeable, although the amount of shifting can not be linearly related with the fading parameter values.

\section{Conclusion}

In this paper, we investigate the energy efficiency of three different relay assisted communication strategies in sensor networks operating over a $\alpha-\mu$ fading channel. The cooperative strategies combine the relayed signal with the direct signal from source through SC or MRC, whereas in the non-cooperative strategy the direct signal is discarded. The results indicate that co-operation does not necessarily yield lower energy consumption, especially for small source destination separations. While comparing the cooperative schemes for different source destination distances, spectral efficiency values, and fading parameters, we found that SC is preferable than MRC as SC is more robust against parameter variations and incurs lower receiver complexity. In addition, the energy saving with MRC is nominal and not guaranteed across the whole range of parameter values (e.g. high $\alpha$ values). Further, the optimal location of the relay is also dependent on the system parameters and may be quite far away from the mid-point between source and destination. This demands careful inspection of all intermediate nodes when being considered for potential candidates as relays in a sensor network.

\section{Acknowledgments}

This work was supported by the SoMoPro II programme, Project No. 3SGA5720, co-financed by the People
Programme (Marie Curie action) of the Seventh Framework Programme of EU according to the REA Grant Agreement No. 291782 and by the South-Moravian Region. This publication reflects only the author's views and the Union is not liable for any use that may be made of the information contained therein.

\section{References}

[1] FENG, D., JIANG, C., LIM, G., et al. A survey of energy-efficient wireless communications. IEEE Communications Surveys \& Tutorials, 2013, vol. 15, no. 1, p. 167-178. DOI: $10.1109 /$ SURV.2012.020212.00049

[2] ZHANG, Z., WANG, J., WU, Q. ARQ protocols in cognitive decodeand-forward relay networks: Opportunities gain. Radioengineering, 2015, vol. 24, no. 1, p. 296-304. DOI: 10.13164/re.2015.0296

[3] MORAES, A. C., DA COSTA, D. B., YACOUB, M. D. An outage analysis of multibranch diversity receivers with cochannel interference in $\alpha-\mu, \kappa-\mu$, and $\eta-\mu$ fading scenarios. Wireless Personal Communications, 2012, vol. 64, no. 1, p. 3-19. DOI: 10.1007/s11277012-0513-x

[4] YACOUB, M. D. The $\alpha-\mu$ distribution: A physical fading model for the Stacy distribution. IEEE Transactions on Vehicular Technology, 2007, vol. 56, no. 1, p. 27-34. DOI: 10.1109/TVT.2006.883753

[5] GHOSH, B., GHOSH, A., BISWAS, N., et al. Placing the 'third' node: An energy efficiency perspective. In Proceedings of the 5th International Conference on Computers and Devices for Communication (CODEC). Kolkata (India), 2012, p. 1-4. DOI: 10.1109/CODEC.2012.6509228

[6] CHANDRA, A., GHOSH, B., BISWAS, N., et al. Energy efficient relay placement for dual hop wireless transmission. International Journal of Electronics Letters, 2013, vol. 1, no. 4, p. 198-209. DOI: $10.1080 / 21681724.2013 .853370$

[7] CHANDRA, A., BISWAS, S., GHOSH, B., et al. Energy efficient relay placement in dual hop 802.15.4 networks. Wireless Personal Communications, 2014, vol. 75, no. 4, p. 1947-1967. DOI: $10.1007 / \mathrm{s} 11277-013-1447-7$

[8] CUI, S., GOLDSMITH, A., BAHAI, A. Energy-efficiency of MIMO and cooperative MIMO techniques in sensor networks. IEEE Journal on Selected Areas in Communications, 2004, vol. 22, no. 6, p. 1089-1098. DOI: 10.1109/JSAC.2004.830916

[9] WANG, S., NIE, J. Energy efficiency optimization of cooperative communication in wireless sensor network. EURASIP Journal on Wireless Communications and Networking, 2010, vol. 2010, no. 162326 , p. 1-8. DOI: 10.1155/2010/162326

[10] ZHANG, R., GORCE, J.-M. Optimal transmission range for minimum energy consumption in wireless sensor networks. In Proceedings of the IEEE Wireless Communications and Networking Conference (WCNC). Las Vegas (USA), 2008, p. 757-762. DOI: 10.1109/WCNC.2008.139

[11] DE OLIVEIRA BRANTE, G. G., KAKITANI, M. T., SOUZA, R. D. Energy efficiency analysis of some cooperative and non-cooperative transmission schemes in wireless sensor networks. IEEE Transactions on Communications, 2011, vol. 59, no. 10, p. 2671-2677. DOI: 10.1109/TCOMM.2011.063011.100744 
[12] SOUZA, R., KAKITANI, M., BRANTE, G. Energy efficiency analysis of a two dimensional cooperative wireless sensor network with relay selection. Radioengineering, 2013, vol. 22, no. 2, p. 549-557. ISSN: $1210-2512$

[13] SADEK, A. K., YU, W., LIU, K. J. R. On the energy efficiency of cooperative communications in wireless sensor networks. ACM Transactions on Sensor Networks, 2009, vol. 6, no. 1, p. 1-21. DOI: $10.1145 / 1653760.1653765$

[14] KHORMUJI, M., LARSSON, E. G. Cooperative transmission based on decode-and-forward relaying with partial repetition coding. IEEE Transactions on Wireless Communications, 2009, vol. 8, no. 4, p. 1716-1725. DOI: 10.1109/TWC.2009.070674

[15] DA COSTA, D. B., YACOUB, M. D., SANTOS FILHO, J. C. S. Highly accurate closed-form approximations to the sum of $\alpha-\mu$ variates and applications. IEEE Transactions on Wireless Communications, 2008, vol. 7, no. 9, p. 3301-3306. DOI: 10.1109/TWC.2008.070336

[16] PENA-MARTIN, J., ROMERO-JEREZ, J., TELLEZ-LABAO, D. C. Performance of selection combining diversity in $\eta-\mu$ fading channels with integer values of $\mu$. IEEE Transactions on Vehicular Technology, 2015, vol. 64, no. 2, p. 834-839. DOI: 10.1109/TVT.2014.2326333

[17] BISWAS, N., GHOSH, B., CHANDRA, A. Energy efficiency relay node placement in a eta-mu fading channel. In Proceedings of the IEEE Conference on Information \& Communication Technologies (ICT). Kumaracoil (India), 2013, p. 824-828. DOI: 10.1109/CICT.2013.6558208

[18] GHOSH, B., BISWAS, S., CHANDRA, A., et al. Energy efficiency analysis of cooperative and non-cooperative communication scheme in alpha-mu fading channel. In Proceedings of the Interdisciplinary Scientific e Conference (ISeC). Bratislava (Slovakia), 2015, p. 1-11.

[19] GOLDSMITH, A. Wireless Communications. 2nd ed. New York (USA): Cambridge University Press, 2005. ISBN: 9780521837163

[20] LIN, M., WEI, H., OUYANG, J., et al. Performance analysis of a dual-hop cooperative relay network with co-channel interference. Radioengineering, 2014, vol. 23, no. 4, p. 1234-1240. ISSN: 12102512

\section{About the Authors...}

Biswajit GHOSH was born in Birbhum, West Bengal, India. He received B.Tech. degree in Electronics \& Instrumentation from Asansol Engineering College, WB, India in 2006, M.Tech. in Information Technology from NERIST, AP, India in 2008, and currently pursuing Ph.D. at NIT Durgapur. $\mathrm{He}$ is also holding the position of an Assistant Professor at FIEM, Kolkata, WB. His research interests include energy efficiency of cooperative wireless networks.

Sankalita BISWAS was born in Burdwan, West Bengal, India. She received her B.Tech. degree in Electronics \& Communication Engineering from West Bengal University of Technology in 2007, and M.Tech. degree in Electronics and Communication Engineering (Microwave) from Burdwan University, WB in 2010. She is currently working as a full time Ph.D. scholar at NIT Durgapur.

Aniruddha CHANDRA (corresponding author) was born in Suri, West Bengal, India. He received B.E., M.E., and Ph.D. from Jadavpur University, Kolkata, India in 2003, 2005, and 2011 respectively. He joined ECE department, NIT Durgapur in 2005 as an Assistant Professor. From Aug. 2011 to Jan. 2012 he was a Visiting Professor at AIT Bangkok. Currently he is a Marie Curie fellow at Brno University of Technology, Czech Republic. His primary area of research is physical layer issues in wireless communication.

Ashis KUMAR MAL was born in Midnapur, West Bengal, India. He recived B.Tech. degree from Bengal Engg. \& Sc. University, Howrah, India in 1989, and M.Tech. degree from Indian Institute of Technology (IIT)-Kanpur, India in 1994 and Ph.D. from Indian Institute of Technology (IIT)Kharagpur, India. He is currently serving as an Associate Professor in ECE department, NIT Durgapur. 